\title{
Compactness and sequential completeness in some spaces of operators
}

\author{
Marian Nowak
}

Received: 24 December 2012 / Accepted: 10 June 2013 / Published online: 3 July 2013

(C) The Author(s) 2013. This article is published with open access at Springerlink.com

\begin{abstract}
Let $X$ be a completely regular Hausdorff space and $C_{b}(X)$ be the Banach lattice of all real-valued bounded continuous functions on $X$, endowed with the strict topologies $\beta_{\sigma}, \beta_{\tau}$ and $\beta_{t}$. Let $\mathcal{L}_{\beta_{z}, \xi}\left(C_{b}(X), E\right)(z=\sigma, \tau, t)$ stand for the space of all $\left(\beta_{z}, \xi\right)$-continuous linear operators from $C_{b}(X)$ to a locally convex Hausdorff space $(E, \xi)$, provided with the topology $\mathcal{T}_{s}$ of simple convergence. We characterize relative $\mathcal{T}_{s}$-compactness in $\mathcal{L}_{\beta_{z}, \xi}\left(C_{b}(X), E\right)$ in terms of the representing Baire vector measures. It is shown that if $(E, \xi)$ is sequentially complete, then the spaces $\left(\mathcal{L}_{\beta_{z}, \xi}\left(C_{b}(X), E\right), \mathcal{T}_{s}\right)$ are sequentially complete whenever $z=\sigma ; z=\tau$ and $X$ is paracompact; $z=t$ and $X$ is paracompact and Čech complete. Moreover, a Dieudonné-Grothendieck type theorem for operators on $C_{b}(X)$ is given.
\end{abstract}

Keywords Spaces of bounded continuous functions - Strict topologies - Dini topologies · Continuous linear operators · Topology of simple convergence · Baire measures $\cdot$ Banach lattice $\cdot$ Compactness $\cdot$ Sequential completeness

Mathematics Subject Classification (2010) $\quad 46 \mathrm{G} 10 \cdot 28 \mathrm{~A} 32 \cdot 28 \mathrm{~A} 25 \cdot 46 \mathrm{~A} 70$

\section{Introduction and terminology}

For terminology concerning vector lattices we refer the reader to [1]. We denote by $\sigma(L, K), \tau(L, K)$ and $\beta(L, K)$ the weak topology, the Mackey topology and the strong topology on $L$, with respect to a dual pair $\langle L, K\rangle$.

M. Nowak $(\varangle)$

Faculty of Mathematics, Computer Science and Econometrics, University of Zielona Góra, ul. Szafrana 4A, 65516 Zielona Góra, Poland e-mail: M.Nowak@wmie.uz.zgora.pl 
From now on we assume that $X$ is a completely regular Hausdorff space. Let $C_{b}(X)$ be the Banach lattice of all real-valued bounded continuous functions on $X$, endowed with the uniform norm $\|\cdot\|$. Then the Banach dual $C_{b}(X)^{\prime}$ of $C_{b}(X)$ with the natural order $\left(\Phi_{1} \leq \Phi_{2}\right.$ if $\Phi_{1}(u) \leq \Phi_{2}(u)$ for each $\left.0 \leq u \in C_{b}(X)\right)$ is a Dedekind complete Banach lattice. By $C_{b}(X)^{\prime \prime}$ we will denote the Banach bidual of $C_{b}(X)$.

Let $\mathcal{B}$ be the algebra of Baire sets in $X$, which is the algebra generated by the class $\mathcal{Z}$ of all zero-sets of functions of $C_{b}(X)$. Let $M(X)$ stand for the space of all Baire measures on $\mathcal{B}$. Then $M(X)$ with the norm $\|\mu\|=|\mu|(X)$ (= the total variation of $\mu$ ) and the natural order $\left(\mu_{1} \leq \mu_{2}\right.$ if $\mu_{1}(A) \leq \mu_{2}(A)$ for all $\left.A \in \mathcal{B}\right)$ is a Dedekind complete Banach lattice (see [20, p. 114, p. 122]). Due to the Alexandrov representation theorem (see [19], [20, Theorem 5.1]) $C_{b}(X)^{\prime}$ can be identified with $M(X)$ through the lattice isomorphism $M(X) \ni \mu \mapsto \Phi_{\mu} \in C_{b}(X)^{\prime}$, where $\Phi_{\mu}(u)=\int_{X} u d \mu$ for all $u \in C_{b}(X)$, and $\left\|\Phi_{\mu}\right\|=\|\mu\|$.

The strict topologies $\beta_{\sigma}, \beta_{\tau}$ and $\beta_{t}$ on $C_{b}(X)$ are of importance in the topological measure theory (see [18], [20] for more details). Note that in [18] $\beta_{\sigma}, \beta_{\tau}, \beta_{t}$ are denoted by $\beta_{1}, \beta, \beta_{0}$ respectively. It is well known that $\beta_{z}(z=\sigma, \tau, t)$ is a locally convex-solid topology (see [20, Theorem 11.6]), and $\beta_{t} \subset \beta_{\tau} \subset \beta_{\sigma} \subset \mathcal{T}_{\|\cdot\|}$. Recall that $\beta_{\sigma}$ is a $\sigma$-Dini topology (resp. $\beta_{\tau}$ is a Dini topology), that is $u_{n} \rightarrow 0$ in $\beta_{\sigma}$ whenever $u_{n}(x) \downarrow 0$ for all $x \in X$ (resp. $u_{\alpha} \rightarrow 0$ in $\beta_{\tau}$ whenever $u_{\alpha}(x) \downarrow 0$ for all $x \in X$ ) (see [18, Theorem 6.2], [20, Theorems 11.16 and 11.28]). $\beta_{t}$ is the finest locally convex topology on $C_{b}(X)$ that agrees with the compact-open topology $\eta$ on each set $B_{r}=\left\{u \in C_{b}(X),\|u\| \leq r\right\}, r>0$ (see [20, Theorem 10.5]). Moreover, $\left(C_{b}(X), \beta_{z}\right.$ ) (for $z=\sigma ; z=\tau$ whevener $X$ is paracompact; $z=t$ whenever $X$ is paracompact and Čech complete) is a strongly Mackey space, that is, every relatively countably $\sigma\left(C_{b}(X)_{\beta_{z}}^{\prime}, C_{b}(X)\right)$-compact subset of $C_{b}(X)_{\beta_{z}}^{\prime}$ is $\beta_{z}$-equicontinuous (see [20, Theorems 11.5, 12.22 and 12.9], [18, Theorem 4.5]). We have (see [20, Theorem 11.8], [18, Theorem 4.3]):

$$
\left(C_{b}(X), \beta_{z}\right)^{\prime}=\left\{\Phi_{\mu}: \mu \in M_{z}(X)\right\}=L_{z}\left(C_{b}(X)\right)(z=\sigma, \tau, t),
$$

where $M_{\sigma}(X), M_{\tau}(X), M_{t}(X)$ are subspaces of $M(X)$ of all $\sigma$-additive $\tau$-additive and tight Baire measures, respectively. $L_{\sigma}\left(C_{b}(X)\right), L_{\tau}\left(C_{b}(X)\right)$ and $L_{t}\left(C_{b}(X)\right)$ are subspaces of $C_{b}(X)^{\prime}$ of all $\sigma$-additive, $\tau$-additive and tight functionals, respectively.

From now on we assume that $(E, \xi)$ is a locally convex Hausdorff space (briefly, lcHs). Let $\mathcal{P}_{\xi}$ stand for a directed family of seminorms on $E$ that generates $\xi$.

Following the definitions of $\sigma$-additive, $\tau$-additive and tight functionals on $C_{b}(X)$ one can distinguish the corresponding classes of linear operators on $C_{b}(X)$.

Definition 1.1 A linear operator $T: C_{b}(X) \rightarrow E$ is said to be:

(i) $\sigma$-additive if $T\left(u_{n}\right) \rightarrow 0$ for $\xi$ whenever $\left(u_{n}\right)$ is a sequence in $C_{b}(X)$ such that $u_{n}(x) \downarrow 0$ for all $x \in X$.

(ii) $\tau$-additive if $T\left(u_{\alpha}\right) \rightarrow 0$ for $\xi$ whenever $\left(u_{\alpha}\right)$ is a net in $C_{b}(X)$ such that $u_{\alpha}(x) \downarrow$ 0 for all $x \in X$.

(iii) tight if $T\left(u_{\alpha}\right) \rightarrow 0$ for $\xi$ whenever $\sup _{\alpha}\left\|u_{\alpha}\right\|<\infty$ and $u_{\alpha} \rightarrow 0$ uniformly on compact sets in $X$. 
By $\mathcal{L}_{\|\cdot\|, \xi}\left(C_{b}(X), E\right)$ (resp. $\mathcal{L}_{\beta_{z}, \xi}\left(C_{b}(X), E\right)$ for $\left.z=\sigma, \tau, t\right)$ we will denote the space of all $(\|\cdot\|, \xi)$-continuous (resp. $\left(\beta_{z}, \xi\right)$-continuous) linear operators $T: C_{b}(X) \rightarrow E$. Let $W\left(C_{b}(X), E\right)$ be the space of all weakly compact operators from the Banach space $C_{b}(X)$ to $(E, \xi)$. Then

$$
\mathcal{L}_{\beta_{t}, \xi}\left(C_{b}(X), E\right) \subset \mathcal{L}_{\beta_{\tau}, \xi}\left(C_{b}(X), E\right) \subset \mathcal{L}_{\beta_{\sigma}, \xi}\left(C_{b}(X), E\right) \subset \mathcal{L}_{\|\cdot\|, \xi}\left(C_{b}(X), E\right)
$$

and

$$
W\left(C_{b}(X), E\right) \subset \mathcal{L}_{\|\cdot\|, \xi}\left(C_{b}(X), E\right)
$$

By $\mathcal{T}_{s}$ we will denote the topology of simple convergence on $\mathcal{L}_{\|\cdot\|, \xi}\left(C_{b}(X), E\right)$. Then $\mathcal{T}_{s}$ is generated by the family $\left\{q_{p, u}: p \in \mathcal{P}_{\xi}, u \in C_{b}(X)\right\}$ of seminorms, where

$$
q_{p, u}(T):=p(T(u)) \text { for } T \in \mathcal{L}_{\|\cdot\|, \xi}\left(C_{b}(X), E\right) .
$$

Graves and Ruess [6, Theorem 7] characterized relative compactness in $c a(\Sigma, E)$ (= the space of all $E$-valued countably additive measures on a $\sigma$-algebra $\Sigma$ ) in the topology $\mathcal{T}_{S}$ of simple convergence (convergence on each $A \in \Sigma$ ) in terms of the properties of the integration operators from $\mathcal{S}(\Sigma)$ to $E$ and from $L(\Sigma)$ to $E$. In [12, Theorem 3.2] (resp. [14, Theorem 3.4]) we study relative $\mathcal{T}_{s}$-compactness in the space $\mathcal{L}_{\tau, \xi}(B(\Sigma), E)$ of all $(\tau(B(\Sigma), c a(\Sigma)), \xi)$-continuous linear operators from $B(\Sigma)$ to $E$ (resp. in the space $\mathcal{L}_{\tau, \xi}\left(L^{\infty}(\mu), E\right)$ of all $\left(\tau\left(L^{\infty}(\mu), L^{1}(\mu)\right), \xi\right)$-continuous linear operators from $L^{\infty}(\mu)$ to $E$ ).

In this paper we study topological properties of the spaces $\left.\mathcal{L}_{\beta_{z}, \xi}\left(C_{b}(X), E\right), \mathcal{T}_{s}\right)$ for $z=\sigma, \tau, t$. We characterize relative $\mathcal{T}_{s}$-compactness in $\mathcal{L}_{\beta_{z}, \xi}\left(C_{b}(X), E\right)$ in terms of the corresponding Baire and Borel vector measures (see Theorems 3.2, 4.2, and 5.7 below). It is shown that if $(E, \xi)$ is a sequentially complete lcHs, then the space $\left(\mathcal{L}_{\beta_{z}, \xi}\left(C_{b}(X), E\right), \mathcal{T}_{s}\right)$ is sequentially complete whenever $z=\sigma ; z=\tau$ and $X$ is paracompact; $z=t$ and $X$ is paracompact and Čech complete (see Corollaries 3.4, 4.5 and 5.4 below). Moreover, we derive a Dieudonné-Grothendieck type theorem for tight and weakly compact operators on $C_{b}(X)$ (see Theorem 5.8 below).

\section{Representation of continuous operators on $C_{b}(X)$}

Let $B(\mathcal{B})$ denote the Banach lattice of all functions $u: X \rightarrow \mathbb{R}$ that are uniform limits of sequences of $\mathcal{B}$-simple functions, provided with the uniform norm $\|\cdot\|$.

It is well known that $C_{b}(X) \subset B(\mathcal{B})$ (see [2, Lemma 1.2]) and one can embed isometrically $B(\mathcal{B})$ in $C_{b}(X)^{\prime \prime}$ by the mapping $\pi: B(\mathcal{B}) \rightarrow C_{b}(X)^{\prime \prime}$, where for each $u \in B(\mathcal{B})$,

$$
\pi(u)\left(\Phi_{\mu}\right)=\int_{X} u d \mu \text { for all } \mu \in M(X) .
$$


Assume that $(E, \xi)$ is a locally convex Hausdorff space. By $(E, \xi)^{\prime}$ or $E_{\xi}^{\prime}$ we denote the topological dual of $(E, \xi)$. Then the space $E_{\xi}^{\prime \prime}=\left(E_{\xi}^{\prime}, \beta\left(E_{\xi}^{\prime}, E\right)\right)^{\prime}$ is the bidual of $(E, \xi)$. Let $\mathcal{E}_{\xi}$ stand for the set of all $\xi$-equicontinuous subsets of $E_{\xi}^{\prime}$. Note that $\xi$ is the topology of uniform convergence on all sets $\mathcal{A} \in \mathcal{E}_{\xi}$, i.e., $\xi$ is generated by the family of seminorms $\left\{p_{\mathcal{A}}: \mathcal{A} \in \mathcal{E}_{\xi}\right\}$, where

$$
p_{\mathcal{A}}(e)=\sup \left\{\left|e^{\prime}(e)\right|: e^{\prime} \in \mathcal{A}\right\} \text { for } e \in E \text {. }
$$

Let $\xi_{\varepsilon}$ stand for the topology on $E_{\xi}^{\prime \prime}$ of uniform convergence on all sets $\mathcal{A} \in \mathcal{E}_{\xi}$, i.e., $\xi_{\varepsilon}$ is generated by the family of seminorms $\left\{q_{\mathcal{A}}: \mathcal{A} \in \mathcal{E}_{\xi}\right\}$, where

$$
q_{\mathcal{A}}\left(e^{\prime \prime}\right)=\sup \left\{\left|e^{\prime \prime}\left(e^{\prime}\right)\right|: e^{\prime} \in \mathcal{A}\right\} \text { for } e^{\prime \prime} \in E_{\xi}^{\prime \prime},
$$

(see [5, Chapter 8.7]).

Let $i: E \rightarrow E_{\xi}^{\prime \prime}$ stand for the canonical embedding, i.e., $i(e)\left(e^{\prime}\right)=e^{\prime}(e)$ for $e \in E$ and $e^{\prime} \in E_{\xi}^{\prime}$. Moreover, let $j: i(E) \rightarrow E$ denote the left inverse of $i$, that is, $j \circ i=i d_{E}$. Note that $j$ is $\left(\sigma\left(i(E), E_{\xi}^{\prime}\right), \sigma\left(E, E_{\xi}^{\prime}\right)\right)$-continuous.

Assume that $T: C_{b}(X) \rightarrow E$ is $(\|\cdot\|, \xi)$-continuous linear operator. Let $T^{\prime}$ : $E_{\xi}^{\prime} \rightarrow C_{b}(X)^{\prime}$ and $T^{\prime \prime}: C_{b}(X)^{\prime \prime} \rightarrow E_{\xi}^{\prime \prime}$ denote the conjugate and the biconjugate of $T$, respectively. Let

$$
\hat{T}:=T^{\prime \prime} \circ \pi: B(\mathcal{B}) \rightarrow E_{\xi}^{\prime \prime}
$$

Since the topology $\left(\mathcal{T}_{\|\cdot\|_{C_{b}(X)}}\right)_{\varepsilon}$ on $C_{b}(X)^{\prime \prime}$ coincides with $\|\cdot\|_{C_{b}(X)^{\prime \prime}}$-topology, in view of [5, Proposition 8.7.2] $T^{\prime \prime}$ is $\left(\|\cdot\|_{C_{b}(X)^{\prime \prime}}, \xi_{\varepsilon}\right)$-continuous. Then $\hat{T}$ is $\left(\|\cdot\|, \xi_{\varepsilon}\right)$ continuous. For $A \in \mathcal{B}$ let us put

$$
\hat{m}_{T}(A):=\hat{T}\left(\mathbb{1}_{A}\right) .
$$

Then

$$
\hat{m}_{T}: \mathcal{B} \longrightarrow E_{\xi}^{\prime \prime}
$$

is a $\xi_{\varepsilon}$-bounded measure, called the representing measure for $T$. For each $e^{\prime} \in E_{\xi}^{\prime}$ let

$$
\left(\hat{m}_{T}\right)_{e^{\prime}}(A):=\hat{m}_{T}(A)\left(e^{\prime}\right) \text { for all } A \in \mathcal{B} \text {. }
$$

From the general properties of the operator $\hat{T}$ it follows immediately that

$$
\hat{T}\left(C_{b}(X)\right) \subset i(E) \text { and } T(u)=j(\hat{T}(u)) \text { for all } u \in C_{b}(X) .
$$

The next theorem gives a characterization of $(\|\cdot\|, \xi)$-continuous linear operators $T: C_{b}(X) \rightarrow E$ in terms of their representing measures (see [13, Theorem 2.1]).

Theorem 2.1 Let $T: C_{b}(X) \longrightarrow$ E be a $(\|\cdot\|, \xi)$-continuous linear operator. Then the following statements hold: 
(i) $\left(\hat{m}_{T}\right)_{e^{\prime}} \in M(X)$ for each $e^{\prime} \in E_{\xi}^{\prime}$.

(ii) The mapping $E_{\xi}^{\prime} \ni e^{\prime} \mapsto\left(\hat{m}_{T}\right)_{e^{\prime}} \in M(X)$ is $\left(\sigma\left(E_{\xi}^{\prime}, E\right), \sigma\left(M(X), C_{b}(X)\right)\right)$ continuous.

(iii) For each $e^{\prime} \in E_{\xi}^{\prime}$,

$$
\hat{T}(u)\left(e^{\prime}\right)=e^{\prime}(T(u))=\int_{X} u d\left(\hat{m}_{T}\right)_{e^{\prime}} \text { for all } u \in C_{b}(X) .
$$

Conversely, let $\hat{m}: \mathcal{B} \rightarrow E_{\xi}^{\prime \prime}$ be a vector measure satisfying (i) and (ii). Then there exists a unique $(\|\cdot\|, \xi)$-continuous linear operator $T: C_{b}(X) \rightarrow E$ such that (iii) holds and $\hat{m}(A)=\left(T^{\prime \prime} \circ \pi\right)\left(\mathbb{1}_{A}\right)$ for all $A \in \mathcal{B}$.

In consequence, the vector measure $\hat{m}: \mathcal{B} \rightarrow E_{\xi}^{\prime \prime}$ satisfying (i), (ii) and (iii) is uniquely determined by $a(\|\cdot\|, \xi)$-continuous linear operator $T: C_{b}(X) \rightarrow E$.

In view of Theorem 2.1 and (1.1) we have

Corollary 2.2 Let $T: C_{b}(X) \rightarrow E$ be a $(\|\cdot\|, \xi)$-continuous linear operator, and $z=\sigma, \tau, t$. Then for each $e^{\prime} \in E_{\xi}^{\prime}$ the following statements are equivalent:

(i) $e^{\prime} \circ T \in C_{b}(X)_{\beta_{z}}^{\prime}$.

(ii) $\left(\hat{m}_{T}\right)_{e^{\prime}} \in M_{z}(X)$.

Note that a subset $\mathcal{K}$ of $\mathcal{L}_{\beta_{z, \xi}}\left(C_{b}(X), E\right)$ is $\left(\beta_{z}, \xi\right)$-equicontinuous $(z=\sigma, \tau, t)$ if and only if for each $\mathcal{A} \in \mathcal{E}_{\xi}$, the set $\left\{e^{\prime} \circ T: T \in \mathcal{K}, e^{\prime} \in \mathcal{A}\right\}$ in $C_{b}(X)_{\beta_{z}}^{\prime}$ is $\beta_{z}$-equicontinuous.

The following result will be of importance (see [17, Theorem 2]).

Theorem 2.3 Let $\mathcal{K}$ be a $\mathcal{T}_{s}$-compact subset of $\mathcal{L}_{\beta_{z}, \xi}\left(C_{b}(X), E\right)$ for $z=\sigma, \tau$, t. If $C$ is a $\sigma\left(E_{\xi}^{\prime}, E\right)$-closed and $\xi$-equicontinuous subset of $E_{\xi}^{\prime}$, then $\left\{e^{\prime} \circ T: T \in \mathcal{K}, e^{\prime} \in C\right\}$ is a $\sigma\left(C_{b}(X)_{\beta_{z}}^{\prime}, C_{b}(X)\right)$-compact set in $C_{b}(X)_{\beta_{z}}^{\prime}$.

Assume now that $T: C_{b}(X) \rightarrow E$ is a weakly compact operator, that is, $T$ maps bounded sets in the Banach space $C_{b}(X)$ into relatively $\sigma\left(E, E_{\xi}^{\prime}\right)$-compact sets in $E$ (hence $T$ is (\| \|\|$, \xi)$-continuous). Then by the Gantmacher type theorem (see [5, Corollary 9.3.2]) we have

$$
T^{\prime \prime}\left(C_{b}(X)^{\prime \prime}\right) \subset i(E)
$$

Let us put

$$
\widetilde{T}:=j \circ T^{\prime \prime} \circ \pi: B(\mathcal{B}) \longrightarrow E
$$

and

$$
m_{T}(A):=\widetilde{T}\left(\mathbb{1}_{A}\right) \text { for } A \in \mathcal{B} .
$$


Note that

$$
\widetilde{T}=j \circ \hat{T} \quad \text { and } \quad m_{T}=j \circ \hat{m}_{T}: \mathcal{B} \longrightarrow E \text {. }
$$

Then for each $e^{\prime} \in E_{\xi}^{\prime}$ we have

$$
\left(\hat{m}_{T}\right)_{e^{\prime}}(A)=\left(e^{\prime} \circ m_{T}\right)(A) \text { for each } A \in \mathcal{B} \text {. }
$$

It follows that for each $\mathcal{A} \in \mathcal{E}_{\xi}$ and $A \in \mathcal{B}$ we have

$$
q_{\mathcal{A}}\left(\hat{m}_{T}(A)\right)=p_{\mathcal{A}}\left(m_{T}(A)\right) .
$$

For terminology and basic results concerning integration with respect to vector measures we refer to $[7,10,15,16]$. Recall that a vector measure $m: \mathcal{B} \rightarrow E$ is said to be $\xi$-strongly bounded if $m\left(A_{n}\right) \rightarrow 0$ in $\xi$ for each pairwise disjoint sequence $\left(A_{n}\right)$ in $\mathcal{B}$.

The following Alexandrov type theorem for weakly compact operators on $C_{b}(X)$ is of importance (see [13, Theorems 4.1 and 4.2]).

Theorem 2.4 Assume that $(E, \xi)$ is a quasicomplete lcHs. Then for a weakly compact operator $T: C_{b}(X) \rightarrow E$ the following statements hold:

(i) $m_{T}: \mathcal{B} \rightarrow E$ is $\xi$-strongly bounded.

(ii) $\hat{m}_{T}: \mathcal{B} \rightarrow E_{\xi}^{\prime \prime}$ is $\xi_{\varepsilon}$-strongly bounded.

(iii) $T(u)=\int_{X} u d m_{T}$ for all $u \in C_{b}(X)$.

\section{Topological properties of the space $\mathcal{L}_{\beta_{\sigma}, \xi}\left(C_{b}(X), E\right)$}

We start with a characterization of $\left(\beta_{\sigma}, \xi\right)$-equicontinuous sets in $\mathcal{L}_{\beta_{\sigma}, \xi}\left(C_{b}(X), E\right)$.

Proposition 3.1 For a subset $\mathcal{K}$ of $\mathcal{L}_{\beta_{\sigma}, \xi}\left(C_{b}(X), E\right)$ the following statement are equivalent:

(i) $\mathcal{K}$ is $\left(\beta_{\sigma}, \xi\right)$-equicontinuous.

(ii) $\mathcal{K}$ is uniformly $\sigma$-additive, i.e., $T\left(u_{n}\right) \rightarrow 0$ in $\xi$ uniformly for $T \in \mathcal{K}$ whenever $u_{n}(x) \downarrow 0$ for all $x \in X$.

(iii) The set $\left\{\hat{m}_{T}(A): T \in \mathcal{K}, A \in \mathcal{B}\right\}$ is $\xi_{\varepsilon}$-bounded in $E_{\xi}^{\prime \prime}$ and $\hat{m}_{T}\left(Z_{n}\right) \rightarrow 0$ in $\xi_{\varepsilon}$ uniformly for $T \in \mathcal{K}$ whenever $Z_{n} \downarrow \emptyset, Z_{n} \in \mathcal{Z}$.

Moreover, if $(E, \xi)$ is a quasicomplete lcHs and $\mathcal{K} \subset \mathcal{L}_{\beta_{\sigma}, \xi}\left(C_{b}(X), E\right) \cap$ $W\left(C_{b}(X), E\right)$, then each of the statements (i)-(iii) is equivalent to the following:

(iv) $\int_{X} u_{n} d m_{T} \rightarrow 0$ in $\xi$ uniformly for $T \in \mathcal{K}$ whenever $u_{n}(x) \downarrow 0$ for $x \in X$.

(v) The set $\left\{m_{T}(A): T \in \mathcal{K}, A \in \mathcal{B}\right\}$ is $\xi$-bounded in $E$ and $m_{T}\left(Z_{n}\right) \rightarrow 0$ in $\xi$ uniformly for $T \in \mathcal{K}$ whenever $Z_{n} \downarrow \varnothing, Z_{n} \in \mathcal{Z}$. 
Proof (i) $\Longrightarrow$ (ii) Assume that $\mathcal{K}$ is $\left(\beta_{\sigma}, \xi\right)$-equicontinuous. Let $p \in \mathcal{P}_{\xi}$ and let $\varepsilon>0$ be given. Then there is a $\beta_{\sigma}$-neighborhood $V$ of 0 in $C_{b}(X)$ such that for each $T \in \mathcal{K}$ we have $p(T(u)) \leq \varepsilon$ for all $u \in V$. Assume that $\left(u_{n}\right)$ is a sequence in $C_{b}(X)$ such that $u_{n}(x) \downarrow 0$ for all $x \in X$. Then $u_{n} \rightarrow 0$ for $\beta_{\sigma}$ because $\beta_{\sigma}$ is a $\sigma$-Dini topology. Choose $n_{\varepsilon} \in \mathbb{N}$ such that $u_{n} \in V$ for $n \geq n_{\varepsilon}$. Hence $\sup _{T \in \mathcal{K}} p\left(T\left(u_{n}\right)\right) \leq \varepsilon$ for $n \geq n_{\varepsilon}$.

(ii) $\Longrightarrow$ (iii) Assume that $\mathcal{K}$ is uniformly $\sigma$-additive, and let $\left(u_{n}\right)$ be a sequence in $C_{b}(X)$ such that $u_{n}(x) \downarrow 0$ for all $x \in X$. Then for each $\mathcal{A} \in \mathcal{E}_{\xi}$, we have

$$
\sup _{T \in \mathcal{K}} p_{\mathcal{A}}\left(T\left(u_{n}\right)\right)=\sup _{T \in \mathcal{K}}\left(\sup \left\{\left|e^{\prime}\left(T\left(u_{n}\right)\right)\right|: e^{\prime} \in \mathcal{A}\right\}\right) \rightarrow 0 \text {. }
$$

This means that the set $\left\{e^{\prime} \circ T: T \in \mathcal{K}, e^{\prime} \in \mathcal{A}\right\}$ in $C_{b}(X)_{\beta_{\sigma}}^{\prime}$ is uniformly $\sigma$-additive. Assume that $Z_{n} \downarrow \varnothing, Z_{n} \in \mathcal{Z}$. In view of [20, Theorem 11.14] we get

$$
\sup _{T \in \mathcal{K}} q_{\mathcal{A}}\left(\hat{m}_{T}\left(Z_{n}\right)\right)=\sup _{T \in \mathcal{K}}\left(\sup \left\{\left|\left(\hat{m}_{T}\right)_{e^{\prime}}\left(Z_{n}\right)\right|: e^{\prime} \in \mathcal{A}\right\}\right) \rightarrow 0 .
$$

This means that $\hat{m}_{T}\left(Z_{n}\right) \rightarrow 0$ in $\xi_{\varepsilon}$ uniformly for $T \in \mathcal{K}$. Moreover, we have $\sup \left\{\left|\left(\hat{m}_{T}\right)_{e^{\prime}}(A)\right|: T \in \mathcal{K}, e^{\prime} \in \mathcal{A}, A \in \mathcal{B}\right\} \leq \sup \left\{\left|\left(\hat{m}_{T}\right)_{e^{\prime}}\right|(X): T \in \mathcal{K}, e^{\prime} \in \mathcal{A}\right\}<\infty$.

It follows that

$$
\sup \left\{q_{\mathcal{A}}\left(\hat{m}_{T}(A)\right): T \in \mathcal{K}, A \in \mathcal{B}\right\}<\infty,
$$

i.e., the set $\left\{\hat{m}_{T}(A): T \in \mathcal{K}, A \in \mathcal{B}\right\}$ is $\xi_{\varepsilon}$-bounded in $E_{\xi}^{\prime \prime}$.

(iii) $\Longrightarrow$ (i) Assume that $\left\{\hat{m}_{T}(A): T \in \mathcal{K}, A \in \mathcal{B}\right\}$ is $\xi_{\varepsilon}$-bounded in $E_{\xi}^{\prime \prime}$ and $\hat{m}_{T}\left(Z_{n}\right) \rightarrow 0$ in $\xi_{\varepsilon}$ uniformly for $T \in \mathcal{K}$ whenever $Z_{n} \downarrow \emptyset, Z \in \mathcal{Z}$. It follows that for each $\mathcal{A} \in \mathcal{E}_{\xi}$, we have

$$
\begin{aligned}
& \sup \left\{\left|\left(\hat{m}_{T}\right)_{e^{\prime}}\right|(X): T \in \mathcal{K}, e^{\prime} \in \mathcal{A}\right\} \leq 4 \sup \left\{\left|\left(\hat{m}_{T}\right)_{e^{\prime}}(A)\right|: T\right. \\
& \left.\quad \in \mathcal{K}, e^{\prime} \in \mathcal{A}, A \in \mathcal{B}\right\}<\infty
\end{aligned}
$$

and moreover, for each sequence $\left(Z_{n}\right)$ in $\mathcal{Z}$ such that $Z_{n} \downarrow \varnothing$, we have

$$
\sup _{T \in \mathcal{K}} q_{\mathcal{A}}\left(\hat{m}_{T}\left(Z_{n}\right)\right) \rightarrow 0, \quad \text { i.e., } \quad \sup \left\{\left|\left(\hat{m}_{T}\right)_{e^{\prime}}\left(Z_{n}\right)\right|: T \in \mathcal{K}, e^{\prime} \in \mathcal{A}\right\} \rightarrow 0 .
$$

By [20, Theorem 11.14], we obtain that the set $\left\{e^{\prime} \circ T: T \in \mathcal{K}, e^{\prime} \in \mathcal{A}\right\}$ in $C_{b}(X)_{\beta_{\sigma}}^{\prime}$ is $\beta_{\sigma}$-equicontinuous. This means that the set $\mathcal{K}$ is $\left(\beta_{\sigma}, \xi\right)$-equicontinuous.

Assume that $(E, \xi)$ is a quasicomplete $1 \mathrm{cHs}$ and $\mathcal{K}$ is a subset of $\mathcal{L}_{\beta_{\sigma}, \xi}\left(C_{b}(X), E\right) \cap$ $W\left(C_{b}(X), E\right)$. Then in view of (2.1) and Theorem 2.4 we obtain that (ii) $\Longleftrightarrow$ (iv) and (iii) $\Longleftrightarrow(v)$.

Now we can state a characterization of relatively $\mathcal{T}_{s}$-compact sets in the space $\mathcal{L}_{\beta_{\sigma}, \xi}\left(C_{b}(X), E\right)$. 
Theorem 3.2 Let $\mathcal{K}$ be a subset of $\mathcal{L}_{\beta_{\sigma}, \xi}\left(C_{b}(X), E\right)$. Then the following statements are equivalent:

(i) $\mathcal{K}$ is relatively $\mathcal{T}_{S}$-compact.

(ii) $\mathcal{K}$ is $\left(\beta_{\sigma}, \xi\right)$-equicontinuous and for each $u \in C_{b}(X)$, the $\operatorname{set}\{T(u): T \in \mathcal{K}\}$ is relatively $\xi$-compact in $E$.

(iii) $\mathcal{K}$ is uniformly $\sigma$-additive and for each $u \in C_{b}(X)$, the set $\{T(u): T \in \mathcal{K}\}$ is relatively $\xi$-compact in $E$.

(iv) The following conditions hold:

(a) $\left\{\hat{m}_{T}(A): T \in \mathcal{K}, A \in \mathcal{B}\right\}$ is $\xi_{\varepsilon}$-bounded in $E_{\xi}^{\prime \prime}$.

(b) $\hat{m}_{T}\left(Z_{n}\right) \rightarrow 0$ in $\xi_{\varepsilon}$ uniformly for $T \in \mathcal{K}$ whenever $Z_{n} \downarrow \varnothing, Z_{n} \in \mathcal{Z}$.

(c) For each $u \in C_{b}(X)$, the set $\{T(u): T \in \mathcal{K}\}$ is relatively $\xi$-compact in $E$.

Proof (i) $\Longleftrightarrow$ (ii) See [13, Theorem 3.3].

(ii) $\Longleftrightarrow$ (iii) $\Longleftrightarrow$ (iv) It follows from Proposition 3.1.

The following Banach-Steinhaus type theorem for $\sigma$-additive operators $T$ : $C_{b}(X) \rightarrow E$ will be useful (see [13, Corollary 3.7]).

Proposition 3.3 Let $T_{n}: C_{b}(X) \rightarrow E$ be $\sigma$-additive operators for $n \in \mathbb{N}$. Assume that $T(u)=\xi-\lim T_{n}(u)$ exists for all $u \in C_{b}(X)$. Then

(i) $T: C_{b}(X) \rightarrow E$ is a $\sigma$-additive operator.

(ii) The family $\left\{T_{n}: n \in \mathbb{N}\right\}$ is uniformly $\sigma$-additive.

As a consequence of Proposition 3.3 we get:

Corollary 3.4 Assume that $(E, \xi)$ is a sequentially complete lcHs. Then the space $\left(\mathcal{L}_{\beta_{\sigma}, \xi}\left(C_{b}(X), E\right), \mathcal{T}_{s}\right)$ is sequentially complete.

Proof Let $\left(T_{n}\right)$ be a $\mathcal{T}_{s}$-Cauchy sequence in $\mathcal{L}_{\beta_{\sigma}, \xi}\left(C_{b}(X), E\right)$. Then for each $u \in$ $C_{b}(X),\left(T_{n}(u)\right)$ is a $\xi$-Cauchy sequence in $E$, and hence $T(u)=\xi-\lim T_{n}(u)$ exists. By Proposition 3.3 the operator $T: C_{b}(X) \rightarrow E$ is $\sigma$-additive, i.e., $T \in$ $\mathcal{L}_{\beta_{\sigma}, \xi}\left(C_{b}(X), E\right)$ and $T_{n} \rightarrow T$ in $\mathcal{T}_{s}$, as desired.

\section{Topological properties of the space $\mathcal{L}_{\beta_{\tau}, \xi}\left(C_{b}(X), E\right)$}

Now arguing as in the proof of Proposition 3.1 and using [20, Theorem 11.24] and the fact that $\beta_{\tau}$ is a Dini topology, we can obtain the following characterization of $\left(\beta_{\tau}, \xi\right)$-continuous subsets of $\mathcal{L}_{\beta_{\tau}, \xi}\left(C_{b}(X), E\right)$.

Proposition 4.1 For a subset $\mathcal{K}$ of $\mathcal{L}_{\beta_{\tau}, \xi}\left(C_{b}(X), E\right)$ the following statements are equivalent:

(i) $\mathcal{K}$ is $\left(\beta_{\tau}, \xi\right)$-equicontinuous.

(ii) $\mathcal{K}$ is uniformly $\tau$-additive, i.e., $T\left(u_{\alpha}\right) \rightarrow 0$ in $\xi$ uniformly for $T \in \mathcal{K}$ whenever $u_{\alpha}(x) \downarrow 0$ for all $x \in X$.

(iii) The set $\left\{\hat{m}_{T}(A): T \in \mathcal{K}, A \in \mathcal{B}\right\}$ is $\xi_{\varepsilon}$-bounded in $E_{\xi}^{\prime \prime}$ and $\hat{m}_{T}\left(Z_{\alpha}\right) \rightarrow 0$ in $\xi_{\varepsilon}$ uniformly for $T \in \mathcal{K}$ whenever $Z_{\alpha} \downarrow \emptyset, Z_{\alpha} \in \mathcal{Z}$. 
Moreover, if $(E, \xi)$ is a quasicomplete lcHs and $\mathcal{K} \subset \mathcal{L}_{\beta_{\tau}, \xi}\left(C_{b}(X), E\right) \cap$ $W\left(C_{b}(X), E\right)$, then each of the statements (i)-(iii) is equivalent to the following:

(iv) $\int_{X} u_{\alpha} d m_{T} \rightarrow 0$ in $\xi$ uniformly for $T \in \mathcal{K}$ whenever $u_{\alpha}(x) \downarrow 0$ for $x \in X$.

(v) The set $\left\{m_{T}(A): T \in \mathcal{K}, A \in \mathcal{B}\right\}$ is $\xi$-bounded in $E$ and $m_{T}\left(Z_{\alpha}\right) \rightarrow 0$ in $\xi$ uniformly for $T \in \mathcal{K}$ whenever $Z_{\alpha} \downarrow \emptyset, Z_{\alpha} \in \mathcal{Z}$.

It is known that if $X$ is paracompact, then $\left(C_{b}(X), \beta_{\tau}\right)$ is a strongly Mackey space (see [20, Theorem 12.22]). Now we are ready to present a characterization of relatively $\mathcal{T}_{s}$-compact sets in the space $\mathcal{L}_{\beta_{\tau}, \xi}\left(C_{b}(X), E\right)$.

Theorem 4.2 Assume that $X$ is paracompact. Then for a subset $\mathcal{K}$ of $\mathcal{L}_{\beta_{\tau}, \xi}\left(C_{b}(X), E\right)$ the following statements are equivalent:

(i) $\mathcal{K}$ is relatively $\mathcal{T}_{S}$-compact.

(ii) $\mathcal{K}$ is $\left(\beta_{\tau}, \xi\right)$-equicontinuous and for each $u \in C_{b}(X)$, the $\operatorname{set}\{T(u): T \in \mathcal{K}\}$ is relatively $\xi$-compact in $E$.

(iii) $\mathcal{K}$ is uniformly $\tau$-additive and for each $u \in C_{b}(X)$, the set $\{T(u): T \in \mathcal{K}\}$ is relatively $\xi$-compact in $E$.

(iv) The following conditions hold:

(a) $\left\{\hat{m}_{T}(A): T \in \mathcal{K}, A \in \mathcal{B}\right\}$ is $\xi_{\varepsilon}$-bounded in $E_{\xi}^{\prime \prime}$.

(b) $\hat{m}_{T}\left(Z_{\alpha}\right) \rightarrow 0$ in $\xi_{\varepsilon}$ uniformly for $T \in \mathcal{K}$ whenever $Z_{\alpha} \downarrow \emptyset, Z_{\alpha} \in \mathcal{Z}$.

(c) For each $u \in C_{b}(X)$, the set $\{T(u): T \in \mathcal{K}\}$ is relatively $\xi$-compact in $E$.

Proof (i) $\Longrightarrow$ (ii) Assume that $\mathcal{K}$ is relatively $\mathcal{T}_{s}$-compact. Let $W$ be an absolutely convex and $\xi$-closed neighborhood of 0 for $\xi$ in $E$. Then the polar $W^{0}$ of $W$ with respect to the dual pair $\left\langle E, E_{\xi}^{\prime}\right\rangle$ is a $\sigma\left(E_{\xi}^{\prime}, E\right)$-closed and $\xi$-equicontinuous subset of $E_{\xi}^{\prime}$ (see [1, Theorem 9.21]). Hence in view of Theorem 2.3 the set $H=\left\{e^{\prime} \circ T: T \in \mathcal{K}, e^{\prime} \in\right.$ $\left.W^{0}\right\}$ in $C_{b}(X)_{\beta_{\tau}}^{\prime}$ is relatively $\sigma\left(C_{b}(X)_{\beta_{\tau}}^{\prime}, C_{b}(X)\right)$-compact. Since $\left(C_{b}(X), \beta_{\tau}\right)$ is a strongly Mackey space, the set $H$ is $\beta_{\tau}$-equicontinuous. It follows that there exists a $\beta_{\tau}$-neighborhood $V$ of 0 in $C_{b}(X)$ such that $H \subset V^{0}$, where $V^{0}$ is the polar of $V$ with respect to the dual pair $\left\langle C_{b}(X), C_{b}(X)_{\beta_{\tau}}^{\prime}\right\rangle$. It follows that for each $T \in \mathcal{K}$ we have that $\left\{e^{\prime} \circ T: e^{\prime} \in W^{0}\right\} \subset V^{0}$, i.e., if $e^{\prime} \in W^{0}$, then $\left|e^{\prime}(T(u))\right| \leq 1$ for all $u \in V$. This means that for each $T \in \mathcal{K}$ we have that $W^{0} \subset T(V)^{0}$. Hence $T(V) \subset T(V)^{00} \subset W^{00}=W$ for each $T \in \mathcal{K}$, i.e., $\mathcal{K}$ is $\left(\beta_{\tau}, \xi\right)$-equicontinuous. Clearly, for each $u \in C_{b}(X)$, the set $\{T(u): T \in \mathcal{K}\}$ is relatively $\xi$-compact in $E$.

(ii) $\Longrightarrow$ (i) It follows from [3, Chap. 3, §3.4, Corollary 1].

(ii) $\Longleftrightarrow$ (iii) $\Longleftrightarrow$ (iv) It follows from Proposition 4.1.

Now we will need the following result.

Proposition 4.3 Assume that $X$ is paracompact. Then for a linear operator $T$ : $C_{b}(X) \rightarrow E$ the following statements are equivalent:

(i) $e^{\prime} \circ T \in L_{\tau}\left(C_{b}(X)\right)$ for each $e^{\prime} \in E_{\xi}^{\prime}$.

(ii) $T$ is $\left(\beta_{\tau}, \xi\right)$-continuous.

(iii) $T$ is $\tau$-additive. 
Proof (i) $\Longrightarrow$ (ii) Assume that $e^{\prime} \circ T \in L_{\tau}\left(C_{b}(X)\right)=C_{b}(X)_{\beta_{\tau}}^{\prime}$ for each $e^{\prime} \in$ $E_{\xi}^{\prime}$. Then $T$ is $\left(\sigma\left(C_{b}(X), M_{\tau}(X)\right), \sigma\left(E, E_{\xi}^{\prime}\right)\right)$-continuous (see [1, Theorem 9.26]). Hence $T$ is $\left(\tau\left(C_{b}(X), M_{\tau}(X)\right), \tau\left(E, E_{\xi}^{\prime}\right)\right)$-continuous (see [1, Ex.11, p. 149]). Since $\beta_{\tau}=\tau\left(C_{b}(X), M_{\tau}(X)\right)$ (see [20, Theorem 12.22]) and $\xi \subset \tau\left(E, E_{\xi}^{\prime}\right), T$ is $\left(\beta_{\tau}, \xi\right)$ continuous.

(ii) $\Longrightarrow$ (iii) Assume that $T$ is $\left(\beta_{\tau}, \xi\right)$-continuous and let $\left(u_{\alpha}\right)$ be a net in $C_{b}(X)$ such that $u_{\alpha}(x) \downarrow 0$ for all $x \in X$. Then $u_{\alpha} \rightarrow 0$ for $\beta_{\tau}$ because $\beta_{\tau}$ is a Dini topology. It follows that $T\left(u_{\alpha}\right) \rightarrow 0$ for $\xi$.

(iii) $\Longrightarrow$ (i) It is obvious.

As a consequence of Proposition 4.3 we can derive the following Banach-Steinhaus type theorem for $\tau$-additive operators $T: C_{b}(X) \rightarrow E$.

Corollary 4.4 Assume that $X$ is paracompact. Let $T_{n}: C_{b}(X) \rightarrow E$ be $\tau$-additive operators for $n \in \mathbb{N}$. Assume that $T(u)=\xi-\lim T_{n}(u)$ exists for all $u \in C_{b}(X)$. Then

(i) $T$ is a $\tau$-additive operator.

(ii) The family $\left\{T_{n}: n \in \mathbb{N}\right\}$ is uniformly $\tau$-additive.

Proof For each $e^{\prime} \in E_{\xi}^{\prime}$ we have $\left(e^{\prime} \circ T\right)(u)=\lim \left(e^{\prime} \circ T_{n}\right)(u)$ for all $u \in C_{b}(X)$, and it follows that $\left(e^{\prime} \circ T_{n}\right)$ is a $\sigma\left(C_{b}(X)_{\beta_{\tau}}^{\prime}, C_{b}(X)\right)$-Cauchy sequence in $C_{b}(X)_{\beta_{\tau}}^{\prime}$. Since $X$ is normal and metacompact (see $[20, \S 2])$, the space $\left(C_{b}(X)_{\beta_{\tau}}^{\prime}, \sigma\left(C_{b}(X)_{\beta_{\tau}}^{\prime}, C_{b}(X)\right)\right)$ is sequentially complete (see [20, Theorem 14.12], [18, Theorem 8.7], [11]). Hence for $e^{\prime} \in E_{\xi}^{\prime}$ there exists $\Phi_{e^{\prime}} \in C_{b}(X)_{\beta_{\tau}}^{\prime}$ such that $\Phi_{e^{\prime}}(u)=\lim \left(e^{\prime} \circ T_{n}\right)(u)$ for all $u \in C_{b}(X)$. It follows that $e^{\prime} \circ T=\Phi_{e^{\prime}} \in C_{b}(X)_{\beta_{\tau}}^{\prime}=L_{\tau}\left(C_{b}(X)\right)$, and by Proposition 4.3 we have that $T$ is $\tau$-additive and $T_{n} \rightarrow T$ for $\mathcal{T}_{s}$. Since $\left\{T_{n}: n \in \mathbb{N}\right\} \cup\{T\}$ is a $\mathcal{T}_{s}$-compact subset of $\mathcal{L}_{\beta_{\tau}, \xi}\left(C_{b}(X), E\right)$, by Theorem 4.2 the set $\left\{T_{n}: n \in \mathbb{N}\right\}$ is uniformly $\tau$-additive.

Corollary 4.5 Assume that $X$ is paracompact and $(E, \xi)$ is a sequentially complete lcHs. Then the space $\left(\mathcal{L}_{\beta_{\tau}, \xi}\left(C_{b}(X), E\right), \mathcal{T}_{s}\right)$ is sequentially complete.

\section{Topological properties of the space $\mathcal{L}_{\beta_{t}, \xi}\left(C_{b}(X), E\right)$}

Recall that $X$ is said to be Čech complete if it is a $G_{\delta}$ subset of its Stone-Čech compactification $\beta X$ (see [20, $\S 2$, p. 106-107]). It is known that if $X$ is paracompact and Čech complete, then the space $\left(C_{b}(X), \beta_{t}\right)$ is strongly Mackey (see [20, Theorem 12.9]). Hence using Theorem 2.3 and arguing as in the proof of Theorem 4.2 , we can state the following characterization of relatively $\mathcal{T}_{s}$-compact sets in $\mathcal{L}_{\beta_{t}, \xi}\left(C_{b}(X), E\right)$.

Theorem 5.1 Assume that $X$ is paracompact and Čech complete. Then for a subset $\mathcal{K}$ of $\mathcal{L}_{\beta_{t}, \xi}\left(C_{b}(X), E\right)$ the following statements are equivalent:

(i) $\mathcal{K}$ is relatively $\mathcal{T}_{s}$-compact.

(ii) $\mathcal{K}$ is $\left(\beta_{t}, \xi\right)$-equicontinuous and for each $u \in C_{b}(X)$, the $\operatorname{set}\{T(u): T \in \mathcal{K}\}$ is relatively $\xi$-compact in $E$. 
We will need the following characterization of $\left(\beta_{t}, \xi\right)$-continuous operators $T$ : $C_{b}(X) \rightarrow E$.

Theorem 5.2 Assume that $X$ is paracompact and $\check{C}$ ech complete. Then for a linear operator $T: C_{b}(X) \rightarrow E$ the following statements are equivalent:

(i) $e^{\prime} \circ T \in L_{t}\left(C_{b}(X)\right)$ for each $e^{\prime} \in E_{\xi}^{\prime}$.

(ii) $T$ is $\left(\beta_{t}, \xi\right)$-continuous.

(iii) $T$ is tight.

Proof (i) $\Longrightarrow$ (ii) Assume that $e^{\prime} \circ T \in L_{t}\left(C_{b}(X), E\right)=C_{b}(X)_{\beta_{t}}^{\prime}$ for each $e^{\prime} \in E_{\xi}^{\prime}$. Then $T$ is $\left(\sigma\left(C_{b}(X), M_{t}(X)\right), \sigma\left(E, E_{\xi}^{\prime}\right)\right)$-continuous (see [1, Theorem 9.26]). Hence $T$ is $\left(\tau\left(C_{b}(X), M_{t}(X)\right), \tau\left(E, E_{\xi}^{\prime}\right)\right)$-continuous (see [1, Ex. 11, p. 149]). Since $\beta_{t}=$ $\tau\left(C_{b}(X), M_{t}(X)\right)$ and $\xi \subset \tau\left(E, E_{\xi}^{\prime}\right), T$ is $\left(\beta_{t}, \xi\right)$-continuous

(ii) $\Longrightarrow$ (iii) Assume that $T$ is $\left(\beta_{t}, \xi\right)$-continuous, and let $\left(u_{\alpha}\right)$ be a net in $C_{b}(X)$ such that $\sup _{\alpha}\left\|u_{\alpha}\right\|=r<\infty$ and $u_{\alpha} \rightarrow 0$ for the compact-open topology $\eta$ on $C_{b}(X)$. Since $\left.\eta\right|_{B_{r}}=\left.\beta_{t}\right|_{B_{r}}\left(B_{r}=\left\{u \in C_{b}(X):\|u\| \leq r\right\}\right)$, we have that $u_{\alpha} \rightarrow 0$ for $\beta_{t}$. Hence $T\left(u_{\alpha}\right) \rightarrow 0$ for $\xi$.

(iii) $\Longrightarrow$ (i) It is obvious.

It is known that if $X$ is paracompact, then $X$ is metacompact and normal (see $[20, \S 2])$. Hence in view of ([20, Theorem 14.12], [11]), we conclude that if $X$ is paracompact and Čech complete, then the space $\left(C_{b}(X)_{\beta_{t}}^{\prime}, \sigma\left(C_{b}(X)_{\beta_{t}}^{\prime}, C_{b}(X)\right)\right)$ is sequentially complete. Now we can state the following Banach-Steinhaus type theorem for tight operators $T: C_{b}(X) \rightarrow E$.

Corollary 5.3 Assume that $X$ is paracompact and Čech complete. Let $T_{n}: C_{b}(X) \rightarrow$ $E$ be tight operators for $n \in \mathbb{N}$. Assume that $T(u)=\xi-\lim T_{n}(u)$ exists for all $u \in C_{b}(X)$. Then

(i) $T$ is a tight operator.

(ii) The family $\left\{T_{n}: n \in \mathbb{N}\right\}$ is uniformly tight, i.e., $T_{n}\left(u_{\alpha}\right) \underset{\alpha}{\longrightarrow} 0$ in $\xi$ uniformly for $n \in \mathbb{N}$ whenever $\sup _{\alpha}\left\|u_{\alpha}\right\|<\infty$ and $u_{\alpha} \rightarrow 0$ uniformly on compact sets in $X$.

Proof Arguing as in the Proof of Corollary 4.4 and using Theorem 5.2 we see that $T: C_{b}(X) \rightarrow E$ is a tight operator. Since $\left\{T_{n}: n \in \mathbb{N}\right\} \cup\{T\}$ is a $\mathcal{T}_{s}$-compact subset of $\mathcal{L}_{\beta_{t}, \xi}\left(C_{b}(X), E\right)$, by Theorem 5.1 the family $\left\{T_{n}: n \in \mathbb{N}\right\}$ is $\left(\beta_{t}, \xi\right)$-equicontinuous. Let $p \in \mathcal{P}_{\xi}$ and $\varepsilon>0$ be given. Then there exists a neighborhood $V$ of 0 for $\beta_{t}$ such that $\sup _{n} p\left(T_{n}(u)\right) \leq \varepsilon$ for all $u \in V$. Assume that $\sup _{\alpha}\left\|u_{\alpha}\right\|<\infty$ and $u_{\alpha} \rightarrow 0$ for $\eta$. Then $u_{\alpha} \rightarrow 0$ for $\beta_{t}$, and hence there exists $\alpha_{0}$ such that $u_{\alpha} \in V$ for $\alpha \geq \alpha_{0}$. Hence $\sup _{n} p\left(T_{n}\left(u_{\alpha}\right)\right) \leq \varepsilon$ for $\alpha \geq \alpha_{0}$.

Corollary 5.4 Assume that $X$ is paracompact and $\check{C} e c h$ complete, and $(E, \xi)$ is a sequentially complete lcHs. Then the space $\left(\mathcal{L}_{\beta_{t}, \xi}\left(C_{b}(X), E\right), \mathcal{T}_{s}\right)$ is sequentially complete.

Let $\mathcal{B a}$ (resp. $\mathcal{B o}$ ) denote the $\sigma$-algebra of Baire sets (resp. Borel sets) in $X$. By $B(\mathcal{B} a)$ (resp. $B(\mathcal{B} o)$ ) we denote the Banach lattice of all bounded $\mathcal{B} a$-measurable (resp. Bo-measurable) functions $u: X \rightarrow \mathbb{R}$, provided with the uniform norm $\|\cdot\|$. 
Let $m: \mathcal{B} o \rightarrow E$ be a $\xi$-countably additive measure. For $p \in \mathcal{P}_{\xi}$ we define a semivariation $\|m\|_{p}$ of $m$ by

$$
\|m\|_{p}(A):=\sup \left\{\left|e^{\prime} \circ m\right|(A): e^{\prime} \in V_{p}^{o}\right\} \text { for } A \in \mathcal{B} o
$$

where $V_{p}^{o}$ is the polar of $V_{p}=\{e \in E: p(e) \leq 1\}$ in the duality $\left\langle E, E_{\xi}^{\prime}\right\rangle$.

We say that $m$ is inner regular by compact sets (resp. outer regular by open sets) if for each $A \in \mathcal{B} o, p \in \mathcal{P}_{\xi}$ and $\varepsilon>0$ there exists a compact set $K$ in $X, K \subset A$ such that $\|m\|_{p}(A \backslash K) \leq \varepsilon$ (resp. there exists an open set $U$ in $X, A \subset U$ such that $\left.\|m\|_{p}(U \backslash A) \leq \varepsilon\right)$.

Now we present a characterization of tight and weakly compact operators on $C_{b}(X)$.

Theorem 5.5 Assume that $(E, \xi)$ is a quasicomplete lcHs. Let $T: C_{b}(X) \rightarrow E$ be a weakly compact operator. Then the following statements are equivalent:

(i) $T$ is $\left(\beta_{t}, \xi\right)$-continuous.

(ii) $T$ is tight.

(iii) $e^{\prime} \circ T \in L_{t}\left(C_{b}(X)\right)$ for each $e^{\prime} \in E_{\xi}^{\prime}$.

(iv) $e^{\prime} \circ m_{T} \in M_{t}(X)$ for each $e^{\prime} \in E_{\xi}^{\prime}$.

(v) $m_{T}$ can be uniquely extended to a $\xi$-countably additive Borel measure $\widetilde{m}_{T}$ : $\mathcal{B} o \rightarrow E$ which is inner regular by compact sets and outer regular by open sets, and

$$
T(u)=\int_{X} u d m_{T}=\int_{X} u d \widetilde{m}_{T} \text { for all } u \in C_{b}(X) .
$$

Proof (i) $\Longrightarrow$ (ii) See the proof of implication (i) $\Longrightarrow$ (ii) of Theorem 5.2.

(ii) $\Longrightarrow$ (iii) $\Longrightarrow$ (iv) It is obvious.

(iv) $\Longrightarrow$ (v) Assume that $e^{\prime} \circ m_{T} \in M_{t}(X) \subset M_{\sigma}(X)$ for each $e^{\prime} \in E_{\xi}^{\prime}$. Since $m_{T}$ is $\xi$-strongly bounded and $e^{\prime} \circ m_{T}: \mathcal{B} \rightarrow E$ is countably additive (see [20, p. 118]), by the Kluvanek Extension Theorem (see [9, Theorem of Extension], [15, Corollary 2]) $m_{T}$ can be extended to a $\xi$-countably additive measure $\bar{m}_{T}: \mathcal{B} a \rightarrow E$, The uniqueness of this extension follows from the uniqueness of the extension of $e^{\prime} \circ m_{T}$ from $\mathcal{B}$ to $\mathcal{B} a$ for each $e^{\prime} \in E_{\xi}^{\prime}$ (see [20, §6, pp. 117-118]).

Hence by [8, Theorem 4] $\bar{m}_{T}$ can be uniquely extended to a $\xi$-countably additive Borel measure $\tilde{m}_{T}: \mathcal{B} o \rightarrow E$ which is inner regular by compact sets and outer regular by open sets. Since $C_{b}(X) \subset B(\mathcal{B}) \subset B(\mathcal{B} a) \subset B(\mathcal{B} o)$, we have that

$$
T(u)=\int_{X} u d m_{T}=\int u d \widetilde{m}_{T} \text { for all } u \in C_{b}(X) .
$$

(v) $\Longrightarrow$ (i) It follows from [8, Theorem 4]. 
Now assume that $T: C_{b}(X) \rightarrow E$ is a $\left(\beta_{t}, \xi\right)$-continuous and weakly compact operator. Then by Theorem 5.5, for each $e^{\prime} \in E_{\xi}^{\prime}$ we have

$$
\left(e^{\prime} \circ T\right)(u)=\int_{X} u d\left(e^{\prime} \circ m_{T}\right)=\int_{X} u d\left(\widetilde{e^{\prime} \circ m_{T}}\right)=\int_{X} u d\left(e^{\prime} \circ \widetilde{m}_{T}\right)
$$

for all $u \in C_{b}(X)$, where $\widetilde{e^{\prime} \circ m_{T}}$ denotes the compact-regular Borel measure that uniquely extends a tight Baire measure $e^{\prime} \circ m_{T}$. Hence

$$
e^{\prime} \circ \widetilde{m}_{T}=\widetilde{e^{\prime} \circ m_{T}} \text { for each } e^{\prime} \in E_{\xi}^{\prime} .
$$

Proposition 5.6 Assume that $(E, \xi)$ is a quasicomplete lcHs. For a subset $\mathcal{K}$ of $\mathcal{L}_{\beta_{t}, \xi}\left(C_{b}(X), E\right) \cap W\left(C_{b}(X), E\right)$ the following statements are equivalent:

(i) $\mathcal{K}$ is $\left(\beta_{t}, \xi\right)$-equicontinuous.

(ii) The following conditions hold:

(a) $\sup _{T \in \mathcal{K}}\left\|\tilde{m}_{T}\right\|_{p}(X)<\infty$ for each $p \in \mathcal{P}_{\xi}$.

(b) The family $\left\{\tilde{m}_{T}: T \in \mathcal{K}\right\}$ of Borel measures is uniformly tight (i.e., for each $p \in \mathcal{P}_{\xi}$ and $\varepsilon>0$ there exists a compact set $K$ in $X$ such that $\left.\sup _{T \in \mathcal{K}}\left\|\widetilde{m}_{T}\right\|_{p}(X \backslash K) \leq \varepsilon\right)$.

Proof (i) $\Longrightarrow$ (ii) Assume that $T$ is $\left(\beta_{t}, \xi\right)$-continuous. Let $p \in \mathcal{P}_{\xi}$. Then $V_{p}^{o} \in \mathcal{E}_{\xi}$ and it follows that the set $\left\{e^{\prime} \circ T: T \in \mathcal{K}, e^{\prime} \in V_{p}^{o}\right\}$ in $C_{b}(X)_{\beta_{t}}^{\prime}$ is $\beta_{t}$-equicontinuous. Hence in view of (5.1) and (5.2) by [18, Theorem 5.1] we have that

$$
\sup _{T \in \mathcal{K}}\left\|\widetilde{m}_{T}\right\|_{p}(X)=\sup \left\{\left|e^{\prime} \circ \widetilde{m}_{T}\right|(X): T \in \mathcal{K}, e^{\prime} \in V_{p}^{o}\right\}<\infty,
$$

and the family $\left\{e^{\prime} \circ \widetilde{m}_{T}: T \in \mathcal{K}, e^{\prime} \in V_{p}^{o}\right\}$ of compact regular scalar Borel measures is uniformly tight, i.e., for each $\varepsilon>0$ there exists a compact set $K$ in $X$ such that $\sup \left\{\left|e^{\prime} \circ \widetilde{m}_{T}\right|(X \backslash K): T \in \mathcal{K}, e^{\prime} \in V_{p}^{o}\right\} \leq \varepsilon$. It follows that $\sup _{T \in \mathcal{K}}\left\|\widetilde{m}_{T}\right\|_{p}(X \backslash K) \leq$ $\varepsilon$, as desired.

(ii) $\Longrightarrow$ (i) Assume that (ii) holds. Then for each $p \in \mathcal{P}_{\xi}$ we see that

$$
\sup \left\{\left|e^{\prime} \circ \widetilde{m}_{T}\right|(X): T \in \mathcal{K}, e^{\prime} \in V_{p}^{o}\right\}<\infty
$$

and the family $\left\{e^{\prime} \circ \widetilde{m}_{T}: T \in \mathcal{K}, e^{\prime} \in V_{p}^{o}\right\}$ is uniformly tight. Then by (5.1) and [18, Theorem 5.1], we conclude that the family $\left\{e^{\prime} \circ T: T \in \mathcal{K}, e^{\prime} \in V_{p}^{o}\right\}$ in $C_{b}(X)_{\beta_{t}}^{\prime}$ is $\beta_{t}$-equicontinuous. It follows that the family $\mathcal{K}$ is $\left(\beta_{t}, \xi\right)$-equicontinuous.

As a consequence of Theorem 5.1 and Proposition 5.6 we have:

Theorem 5.7 Assume that $X$ is $\check{C}$ ech complete and paracompact and $(E, \xi)$ is a quasicomplete lcHs. Then for a subset $\mathcal{K}$ of $\mathcal{L}_{\beta_{t}, \xi}\left(C_{b}(X), E\right) \cap W\left(C_{b}(X), E\right)$ the following statements are equivalent:

(i) $\mathcal{K}$ is relatively $\mathcal{T}_{s}$-compact in $\mathcal{L}_{\beta_{t}, \xi}\left(C_{b}(X), E\right)$. 
(ii) $\mathcal{K}$ is $\left(\beta_{t}, \xi\right)$-equicontinuous and for each $u \in C_{b}(X)$, the $\operatorname{set}\left\{\int_{X} u d \widetilde{m}_{T}: T \in \mathcal{K}\right\}$ is relatively $\xi$-compact in $E$.

(iii) The following conditions hold:

(a) $\sup _{T \in \mathcal{K}}\left\|\tilde{m}_{T}\right\|_{p}(X)<\infty$ for each $p \in \mathcal{P}_{\xi}$.

(b) The family $\left\{\widetilde{m}_{T}: T \in \mathcal{K}\right\}$ is uniformly tight.

(c) For each $u \in C_{b}(X)$, the set $\left\{\int_{X} u d \widetilde{m}_{T}: T \in \mathcal{K}\right\}$ is relatively $\xi$-compact in $E$.

Assume that $X$ is locally compact. Then $\beta_{t}$ is the original topology $\beta$ of Buck (see [4]) and is generated by the family of seminorms $\left\{p_{v}: v \in C_{0}(X)\right\}$, where

$$
p_{v}(u)=\sup \{|u(x) v(x)|: x \in X\} \text { for } u \in C_{b}(X)
$$

and $C_{0}(X)$ denotes the space of continuous functions on $X$ vanishing at infinity (see [20, Theorem 10.3] for more details). Then $\beta_{t}=\beta_{\tau}$ (see [20, Theorem 10.14]).

Now we are ready to derive a Dieudonné-Grothendieck type theorem for tight and weakly compact operators on $C_{b}(X)$ (see [16, Chapter 5.2]).

Theorem 5.8 Assume that $X$ is locally compact and $(E, \xi)$ is a quasicomplete lcHs. Let $T_{n}: C_{b}(X) \rightarrow E$ be tight and weakly compact operators for $n \in \mathbb{N}$. Assume that $\xi-\lim \widetilde{m}_{T_{n}}(A)$ exists for each open Baire set $A$. Then

(i) $T(u)=\xi-\lim T_{n}(u)$ exists for each $u \in C_{b}(X)$.

(ii) $T: C_{b}(X) \rightarrow E$ is a tight and weakly compact operator.

Proof In view of [16, Theorem 5.2.23] there exists a unique $\xi$-countably additive measure $\tilde{m}: \mathcal{B} o \rightarrow E$ which is inner regular by compact sets and outer regular by open sets and such that

$$
\int_{X} u d \widetilde{m}=\xi-\lim \int_{X} u d \widetilde{m}_{T_{n}}
$$

for all $u \in B(\mathcal{B} o)$. Let

$$
T_{\widetilde{m}}(u)=\int_{X} u d \widetilde{m} \text { for all } u \in B(\mathcal{B} o) .
$$

Since $\widetilde{m}$ is $\xi$-countably additive, $\widetilde{m}$ is $\xi$-strongly bounded and it follows that the integration operator $T_{\widetilde{m}}: B(\mathcal{B o}) \rightarrow E$ is weakly compact (see [7, Theorem 7]). Define $T=\left.T_{\widetilde{m}}\right|_{C_{b}(X)}: C_{b}(X) \rightarrow E$. Then $T$ is weakly compact, and by Theorem $5.5 T$ is tight, as desired.

Acknowledgments The author wishes to thank the referee for useful suggestions that have improved the paper.

Open Access This article is distributed under the terms of the Creative Commons Attribution License which permits any use, distribution, and reproduction in any medium, provided the original author(s) and the source are credited. 


\section{References}

1. Aliprantis, C.D., Burkinshaw, O.: Positive Operators. Academic Press, New York (1985)

2. Aguayo, J., Sánchez, J.: Weakly compact operators and the strict topologies. Bull. Aust. Math. Soc. 39, 353-359 (1989)

3. Bourbaki, N.: Elements of Mathematics, Topological Vector Spaces, Chap. 1-5. Springer, Berlin (1987)

4. Buck, R.C.: Bounded continuous functions on a locally compact space. Michigan Math. J. 5, 95-104 (1958)

5. Edwards, R.E.: Functional Analysis, Theory and Applications. Holt, Rinehart and Winston, New York (1965)

6. Graves, W.H., Ruess, W.: Compactness in spaces of vector-valued measures and a natural Mackey topology for spaces of bounded measurable functions. Contemp. Math. 2, 189-203 (1980)

7. Hoffmann-Jörgensen, J.: Vector measures. Math. Scand. 28, 5-32 (1971)

8. Khurana, S.S.: Vector measures on topological spaces. Georgian Math. J. 14(4), 687-698 (2007)

9. Kluvanek, I., The extension and closure of vector measures. In: Vector and Operator Valued Measures and Applications (Proceedings of Symposium on Snowbird Resort, Alta, Utah, 1972), pp. 175-198. Academic Press, New York (1973)

10. Lewis, D.R.: Integration with respect to vector measures. Pac. J. Math. 33(1), 157-165 (1970)

11. Moran, W.: Measures on metacompact spaces. Proc. Lond. Math. Soc. 20, 507-524 (1970)

12. Nowak, M.: Vector measures and Mackey topologies. Indag. Math. 23, 113-122 (2012)

13. Nowak, M.: Vector measures and strict topologies. Topol. Appl. 159(5), 1421-1432 (2012)

14. Nowak, M.: Absolutely continuous on function spaces and vector measures. Positivity. doi:10.1007/ s11117-012-0187-3

15. Panchapagesan, T.V.: Applications of a theorem of Grothendieck to vector measures. J. Math. Anal. Appl. 214, 89-101 (1997)

16. Panchapagesan, T.V.: The Bartle-Dunford-Schwartz Integral. Monografie Matematyczne, vol. 69. Birkhäuser, Verlag AG (2008)

17. Schaefer, H., Xiao-Dong, Z.: On the Vitali-Hahn-Saks theorem. In: Operator Theory: Advances and Applications, vol. 75. Birkhäuser, Basel, pp. 289-297 (1995)

18. Sentilles, F.D.: Bounded continuous functions on a completely regular spaces. Trans. Am. Math. Soc. 168, 311-336 (1972)

19. Varadarajan, V.S.: Measures on topological spaces. Mat. Sbornik (N.S.), 55:(97), (1961), 35-100; Am. Math. Soc. Transl. 48(2), 161-228 (1965)

20. Wheeler, R.: A survey of Baire measures and strict topologies. Expositiones Math. 1, 97-190 (1983) 